\title{
Alternative Methods for Forecasting Variations in Hospital Bed Admission
}

\author{
S.Sarifah Radiah Shariff ${ }^{1}$, Mohd Azuan Suhaimi ${ }^{2}$, Siti Meriam Zahari ${ }^{3}$, Zuraidah Derasit ${ }^{4}$ \\ ${ }^{1}$ Malaysia Institute of Transport (MITRANS), Universiti Teknologi MARA Shah Alam, Malaysia \\ ${ }^{2,3,4}$ Centre for Statistics and Decision Science, Faculty of Computer \& Mathematical Sciences, \\ UniversitiTeknologi MARA, 40450 Shah Alam, Selangor, Malaysia
}

\section{Article Info}

Article history:

Received Oct 19, 2017

Revised Dec 27, 2017

Accepted Jan 18, 2018

\section{Keywords:}

Forecasting Variations State Space Model (SSM)

\begin{abstract}
The Malaysian healthcare system is well-being recognized for providing a wide range of access to primary healthcare. The number of hospitals is found to be growing in line with the increase in population. However, overcrowding has become the most common scene that people see in every hospital. The number of patients being admitted may somehow mislead healthcare planners, and thus causing them to underestimate the resources that are required within the hospital. Thus, this study aims to identify better forecasting models for variations in hospital bed admission considering State Space Model (SSM). Data on the admission rate of a state hospital was collected, spanning the period of historical data from 2001 until 2015. The findings indicate that State Space model can outperform common model due to its lower Mean Squared Errors. Female aged between 25 -34 years old are found to be having the highest variation, which could lead to unpredictable in terms of being admitted to hospital.
\end{abstract}

Copyright $(0) 2018$ Institute of Advanced Engineering and Science. All rights reserved.

\section{Corresponding Author:}

S.Sarifah Radiah Shariff

Malaysia Institute of Transport (MITRANS),

Universiti Teknologi MARA Shah Alam, Malaysia

Email: 1sntl@ccu.edu.tw

\section{INTRODUCTION}

Nowadays, in the modern world, people faced the challenges and volatile year for living costs in their daily life. Due on the increasing and higher price of treatment and medicine for healthcare, many shifted to have the treatment in government hospital because of the lower price rates. The increase of those who come to get services from the government hospital is an important issue that can lead to the shortage for the total of bed admission in the government hospital. A rise in disease progression rates also can lead to a tremendous increase in the number of hospital bed admission, resulting in high medical expenses. From dayto-day, the totals of people which are treated at hospital are increasing and it is necessary for Ministry of Health $(\mathrm{MOH})$ to plan carefully about the number of bed admission to avoid congestion and shortage problem in government healthcare facility. Related with the rising in consumer demands, the planning of hospital bed admission is very important to ensure that it gives the positive considerable implications for hospital resource allocation. The hospital planners must creatively adjust the important elements such as hospital beds, staffing levels, medicine usages and other related resources. Hence, it is very important to apply suitable forecasting methods in order to optimally manage hospital bed admissions and other related healthcare services. The good forecasting model will play a significant role in the efficient allocation of resources in healthcare systems with constrained budgets. It also can help the hospital management systems in order to optimally manage patient flow and to improve management strategies, efficiency and safety.

Related with the good forecasting method for forecasting variations in number of hospital bed admission, ARIMA model have been applied in a few of previous studies which focus on bed optimization, bed capacity and bed admission. However, its identification techniques seem difficult and complex in order 
to identify the correct model from the class of possible models. This model also comes up with theoretical model and structural relationships which are not distinct with other simple forecast models. ARIMA models also essentially backward looking and it reasons that it always poor when predicting series with turning points. So, in controlling and optimizing the government expenditure on healthcare system at the public hospitals, there is a need to propose a good forecasting method and its alternatives for the best strategy and planning in estimating the healthcare sources, particularly the variations in number of hospital bed admissions. Past studies that used ARIMA can be found in [1,2,3,4]. Queuing Theory is also applied to predict monthly responsiveness for changing bed related with changing bed demand $[5,6,7,8,9]$. State Space Model (SSM) is known as the model which includes two major elements: an observation process and also a state process. It is known as one model which has powerful framework for the purpose of analysis for the dynamical systems. Hence, this study attempts to apply SSM to forecast variation in number of bed admission.

\section{RESEARCH METHOD}

In state space models, there are three types of inference which always applied for this model which are prediction, filtering and smoothing. In general, the state space model is written as follows:

State equation or transition equation: $\alpha_{t}=\mathbf{T}_{t} \alpha_{t-1}+\mathbf{c}_{\mathbf{t}}+\mathbf{R}_{\mathbf{t}} \boldsymbol{\eta}_{\mathbf{t}}, \boldsymbol{\eta}_{t} \sim \operatorname{iid}\left(0, Q_{t}\right)$

Measurement or observation equation: $\mathbf{y}_{\mathbf{t}}=\mathbf{Z}_{\mathbf{t}} \alpha_{t}+\mathbf{d}_{\mathbf{t}}+\varepsilon_{t}, \quad \varepsilon_{t} \sim \operatorname{iid}\left(0, H_{t}\right)$

Initial state distribution: $\boldsymbol{\alpha}_{0} \sim N\left(\mathbf{a}_{0}, \mathbf{P}_{0}\right)$

where $\mathbf{Z}_{\mathbf{t}}$ is an $\mathrm{Nx}$ m matrix, $\mathbf{d}_{\mathbf{t}}$ is an $\mathrm{Nx} 1$ vector and $\boldsymbol{\varepsilon}_{t}$ is an $\mathrm{Nx} 1$ error vector, $\mathbf{T}_{\mathbf{t}}$ is an $\mathrm{m} \times \mathrm{m}$ transition matrix, $\mathbf{c}_{\mathbf{t}}$ is an $\mathrm{mx} 1$ vector, $\mathbf{R}_{\mathbf{t}}$ is a $\mathrm{mx} g$ matrix, and $\boldsymbol{\eta}_{\mathbf{t}}$ is a $\mathrm{g} \times 1$ error vector. The matrices $\mathbf{Z}_{\mathbf{t}}, \mathbf{d}_{\mathbf{t}}, \mathbf{H}_{\mathbf{t}}, \mathbf{T}_{\mathbf{t}}, \mathbf{c}_{\mathbf{t}}, \mathbf{R}_{\mathbf{t}}$ and $\mathbf{Q}_{t}$ and contains nonrandom elements. The assumptionsof the model are listed as follows:

i) The disturbances of $\varepsilon_{\mathrm{t}}$ and $\eta_{\mathrm{t}}$ are uncorrelated with the initial state variable and also uncorrelated with each other for all time periods.

ii) The initial vector $\boldsymbol{\alpha}_{0}$ has a mean of $\boldsymbol{\alpha}_{0}: \mathrm{E}\left(\boldsymbol{\alpha}_{0}\right)=\boldsymbol{\alpha}_{0}$ and covariance matrix of $\Sigma_{0}: \operatorname{Var}\left(\boldsymbol{\alpha}_{0}\right)=$ $\Sigma_{0}$

iii) The disturbances of $\varepsilon_{\mathrm{t}}$ and $\eta_{\mathrm{t}}$ are normally distributed and serially independent with constant variances.

\subsection{Diagnostic Checks}

All significance test and construction of confidence intervals in State Space Model rely on the assumptions which are related to residual analysis including normality, homoscedasticity and independence. Another diagnostic tool for determining the appropriateness of the model is known as 'auxiliary residuals'. The auxiliary residuals can be applied for the purpose of detecting outliers and structural breaks because $\hat{\varepsilon}_{\mathrm{t}}$ and $\hat{\eta}_{\mathrm{t}}$ are the estimator of $\varepsilon_{\mathrm{t}}$ and $\eta_{\mathrm{t}}$. The smooth observed disturbances of $e_{t}^{*}$ enable for the detection of outlier observations. For smooth state disturbances, it allows the detection of structural breaks in development of time series.

For State Space Model application, packages in R called StructTS and dlm are used. At the same time, a common forecasting model, ARIMA was run to validate the results. ARIMA model was run using Eviews.

\subsection{Data collection}

In this study, hospital bed admission data based on gender and age groups, covering the period of 2001 until 2015 is collected from a state hospital in Malaysia. The age dataset is ranged according to the data source as 0-4, 5-19,20-24, 25-34, 35-44, 45-54, 55-64, 65-74, 75-84, 75+. However, there are missing values of total admission data, particularly during the early years of study duration. To handle the missing data, Amelia II package developed by Professor Gary King from Harvard University is being utilized in the R Development Core Team software package version 3.2.3. The Amelia II R package is an updating version of the Amelia first version. It runs a form of the bootstrap-based EM algorithm to perform the multiple imputation technique that has the ability to compute many more variables that come with many more observations, in considerably faster than existing approaches [10,11]. 


\section{RESULTS AND ANALYSIS}

The results are discussed in several phases: application of models, analysis of variations and performance measurement.

\subsection{Results from ARIMA model}

The result from ARIMA model using Eviews was obtained first in order to check and collect more conclusive evidence about the data stationarity condition. The ACF (Autocorrelation Function) and PACF (Partial Autocorrelation Function) were also plotted to determine the specification of ARIMA model. From Figure 1, the changes vectors of the autocorrelation are decreasing as the time increases, and some even meet to negative values. From the graph, four values of the autocorrelations exceed the significance limit. Hence, there is a need for this series to be made stationary by performing the first difference. Figure 1 shows ACF graph for total of bed admission.

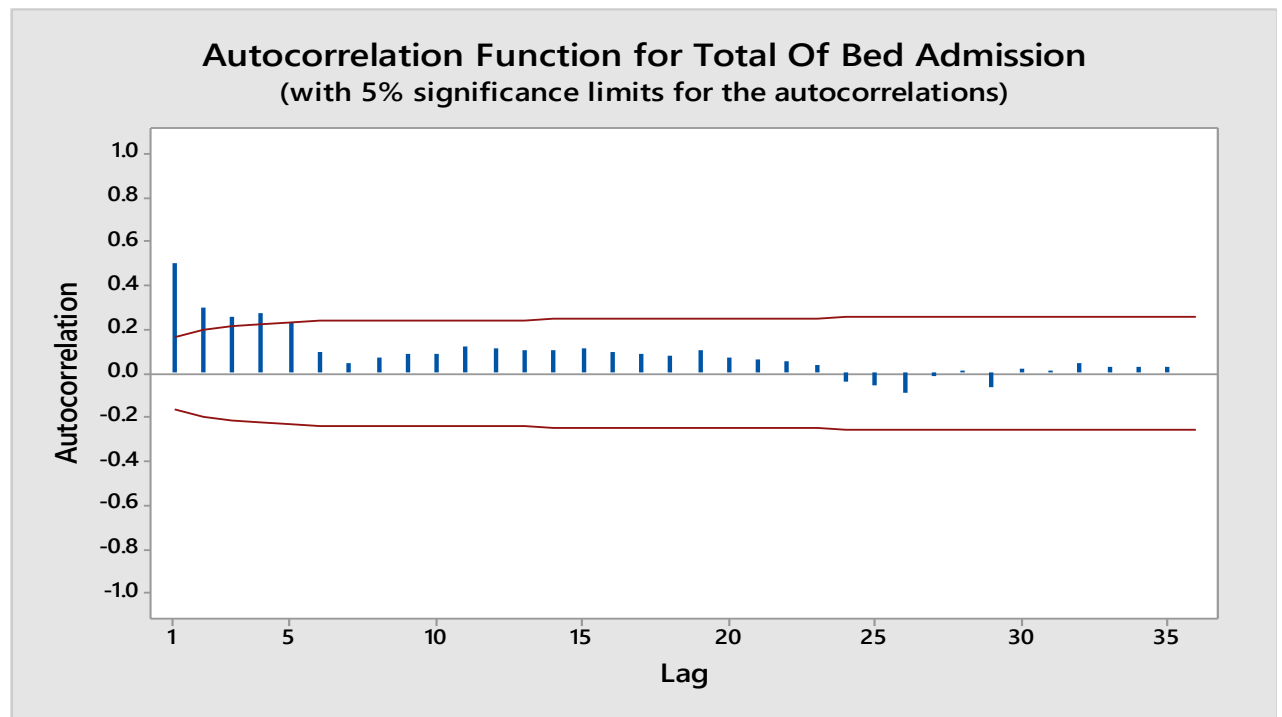

Figure 1. ACF Graph for Total of Bed Admission

\section{Performing First Differencing}

From Figure 2, we can see that the graph showing the dramatic decaying for the data. It is not showing the slowing move up or down from the graph. So, it is concluded that the data now is stationary.

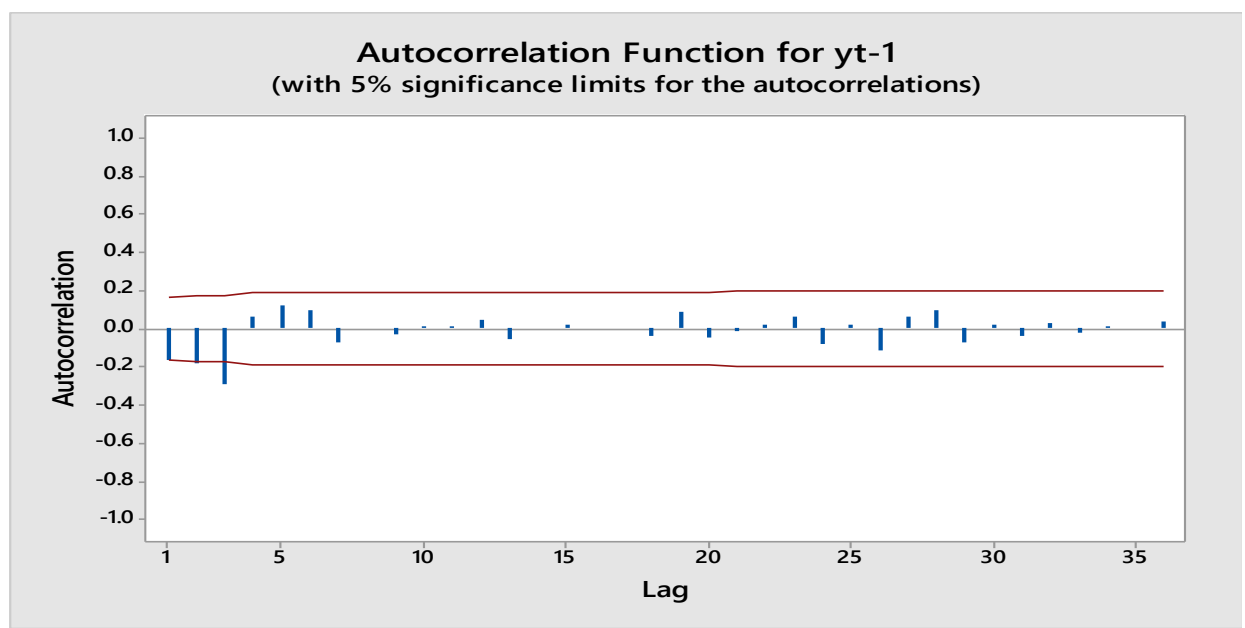

Figure 2. ACF yt-1 Graph 
The data may not perfectly stationary because in economic or business data series such condition may not be easily achievable due to the explainable factors inherent in the data sets.

\section{Model Identification}

In order to identify the best and suitable model, the analysis of the ACF and PACF graphs were done and four models have been identified and estimated using Eviews which are ARIMA $(2,1,2)$, ARIMA $(2,1,0)$, ARIMA $(1,1,1)$ and ARIMA $(1,1,0)$. By comparing the models in Table 1 , the best model is ARIMA $(1,1,1)$ due its smallest MSE $=500372$.

Table 1. Summary of Portmanteau Test

\begin{tabular}{|c|c|c|c|c|}
\hline \multirow{2}{*}{ Statistics } & \multicolumn{4}{|c|}{ Model } \\
\hline & $\operatorname{ARIMA}(2,1,2)$ & $\operatorname{ARIMA}(2,1,0)$ & $\operatorname{ARIMA}(1,1,1)$ & $\operatorname{ARIMA}(1,1,0)$ \\
\hline Calculated Q & 10.2 & 31.3 & 16.1 & 34.6 \\
\hline $\mathrm{DF}$ & 7 & 9 & 9 & 10 \\
\hline Tabulated Q & 14.06 & 16.91 & 16.91 & 18.30 \\
\hline $\begin{array}{c}\text { Decision (5\% sig } \\
\text { level) }\end{array}$ & Accept Ho & Reject Ho & Accept Ho & Reject Ho \\
\hline Conclusion & $\begin{array}{c}\text { The errors are white } \\
\text { noise }\end{array}$ & $\begin{array}{l}\text { The errors are not } \\
\text { white noise }\end{array}$ & $\begin{array}{c}\text { The errors are white } \\
\text { noise }\end{array}$ & $\begin{array}{c}\text { The errors are not } \\
\text { white noise }\end{array}$ \\
\hline MSE & 502283 & 601855 & 500372 & 649141 \\
\hline
\end{tabular}

\subsection{State Space Modelling}

StructTS package in $\mathrm{R}$ is known as Structural time series models in State Space Model, is applied for estimating the parameters of simple State Space Model for each age groups of bed admission. For this study, this package is suitable because the dataset for hospital bed admission is univariate time series and based on 10 age groups based on gender (male and female). It constructs a local linear trend model and estimates the parameters for hospital bed admission for each age groups. Table 2 shows the estimate parameters for local linear trend model for male with 10 age groups for hospital bed admission. From Table 2 , it can be seen that the highest transitional variance (level) values is on age groups 5-19 years old which is 403.70 and for observational variance (epsilon), age groups of 35-44 years old have the highest values with 18808.02 .

The value of transitional variance refers to the maximum likelihood estimates (MLEs) for level, while for observational variance is the value (MLEs) of observation error variances (epsilon). It means that when both of these values are high, there is huge variation in the number of bed being admitted. This is quite critical because the huge variation is critical and will make the forecasting process harder. Huge variation occurs for age group 35-44 years old for male group.

Table 2. Estimate Parameters for Local Linear Trend Model (Male)

\begin{tabular}{|c|c|c|c|c|c|}
\hline Age Groups & $\begin{array}{c}\text { Transitional } \\
\text { variance (level) }\end{array}$ & Slope variance & $\begin{array}{c}\text { Observational } \\
\text { variance (epsilon) }\end{array}$ & $\begin{array}{c}\text { Initial level of } \\
\text { mu }\end{array}$ & $\begin{array}{c}\text { Initial level of } \\
\text { lambda }\end{array}$ \\
\hline Less than 1 & 346.35 & 0 & 712.27 & 154 & 0 \\
\hline $1-4$ & 79.93 & 0 & 382.44 & 97 & 0 \\
\hline $5-19$ & 403.70 & 0 & 3123.73 & 272 & 0 \\
\hline $20-24$ & 144.65 & 0 & 338.13 & 96 & 0 \\
\hline $25-34$ & 97.87 & 0 & 1149.24 & 146 & 0 \\
\hline $35-44$ & 0 & 0 & 18808.02 & 151 & 0 \\
\hline $45-54$ & 90.57 & 0 & 1467.95 & 149 & 0 \\
\hline $55-64$ & 31.87 & 0 & 4004.21 & 183 & 0 \\
\hline $65-74$ & 10.32 & 0 & 5063.44 & 145 & 0 \\
\hline More than 75 & 25.47 & 0 & 1848.63 & 86 & 0 \\
\hline
\end{tabular}

The estimation of parameters for local linear trend model is also done for female which also have 10 age groups. From Table 3, the highest values for transitional variance (level) and also observational variance (epsilon) comes from age group 25-34 years old with values of 2280.85 and 21254.09. It means that the huge variation occurs for this age group. It shows that this age group is more unpredictable in terms of having them being admitted to the hospital. 
Table 3. Estimate Parameters for Local Linear Trend Model (Female)

\begin{tabular}{cccccc}
\hline Age Groups & $\begin{array}{c}\text { Transitional } \\
\text { variance (level) }\end{array}$ & Slope variance & $\begin{array}{c}\text { Observational } \\
\text { variance (epsilon) }\end{array}$ & $\begin{array}{c}\text { Initial level of } \\
\text { mu }\end{array}$ & $\begin{array}{c}\text { Initial level of } \\
\text { lambda }\end{array}$ \\
\hline Less than 1 & 380.85 & 0 & 522.82 & 149 & 0 \\
$1-4$ & 47.33 & 0 & 247.89 & 227 & 0 \\
$5-19$ & 461.40 & 0 & 2214.95 & 336 & 0 \\
$20-24$ & 524.01 & 0 & 3359.41 & 898 & 0 \\
$25-34$ & 2280.85 & 0 & $\mathbf{2 1 2 5 4 . 0 9}$ & 553 & 0 \\
$35-44$ & 1310.96 & 0 & 14712.55 & 149 & 0 \\
$45-54$ & 90.57 & 0 & 867.95 & 77 & 0 \\
$55-64$ & 75.84 & 0 & 3121.52 & 56 & 0 \\
$65-74$ & 15.36 & 0 & 882.25 & & 0 \\
More than 75 & 9.26 & 0 & &
\end{tabular}

\section{Diagnostic for StructTS}

Diagnostic plots for StructTS is a generic function that plots the standardized residuals, autocorrelation function of the residuals and p-values of a Portmanteau test for all lags in datasets hospital bed admission. Based on the diagnostic plots for each age group, there are some age groups that show the same variation in bed admission. For age groups less than one year old, there is huge variation in bed admission during 2002-2005. However, it shows the stable variation until 2014. The same pattern is also seen for several age groups except for (35-44), (45-54), (55-64) and age more than 75 years old.

For age group of (35-44), (45-54) and (55-64) years old, it shows the different variation from the diagnostic plots. The pattern shows the high variation at 2001 and turn to small variation towards the later years. Figure 3 shows the diagnostic plots for these age groups:

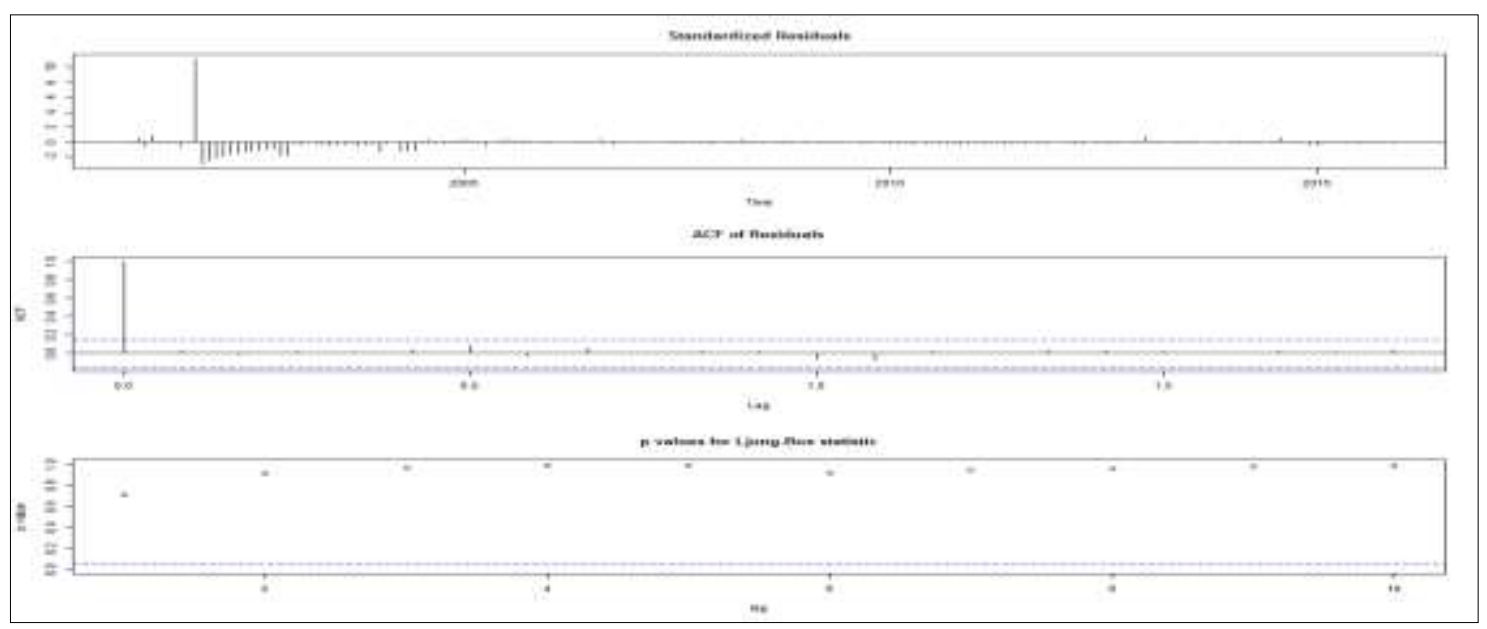

Figure 3. Diagnostic Plots for Age (35-44), (45-54) and (55-64) Years Old

For age groups of (65-74) and age more than 75 years old, the small variation exists at the early years and turn to be high at 2006. However, it is a bit stable up until 2015. Diagnostic plots for female age group also plotted in order to see the differences of variation with male age groups. Similar with male age groups, there are several of female age groups shows the same variation. Figure 4 shows the diagnostics plots for age less than one years old and this variation also same with age groups 20-24 and 25-34 years old. There is small variation in bed admission during 2001 and changes to huge variation at 2002-2004 and it looks big stable up until 2012. However, it shows huge variation again at 2013 towards the later years.

For age group 1-4, 5-19 and 55-64 years old, the pattern shows very small variation at 2001 before turning into huge variation at 2002-2004 and looks big stable up until 2011. However, there is huge variation again at 2012 towards the later years. For age group 35-44 and 45-54 years old the pattern shows high variation in 2001 until 2004 before turning to small variation towards the later years. However, it shows the huge variation again at 2014 towards the later years. At the same time, pattern for female age group of (6574 ) and age more than 75 years old are similar with male group with same ages. 


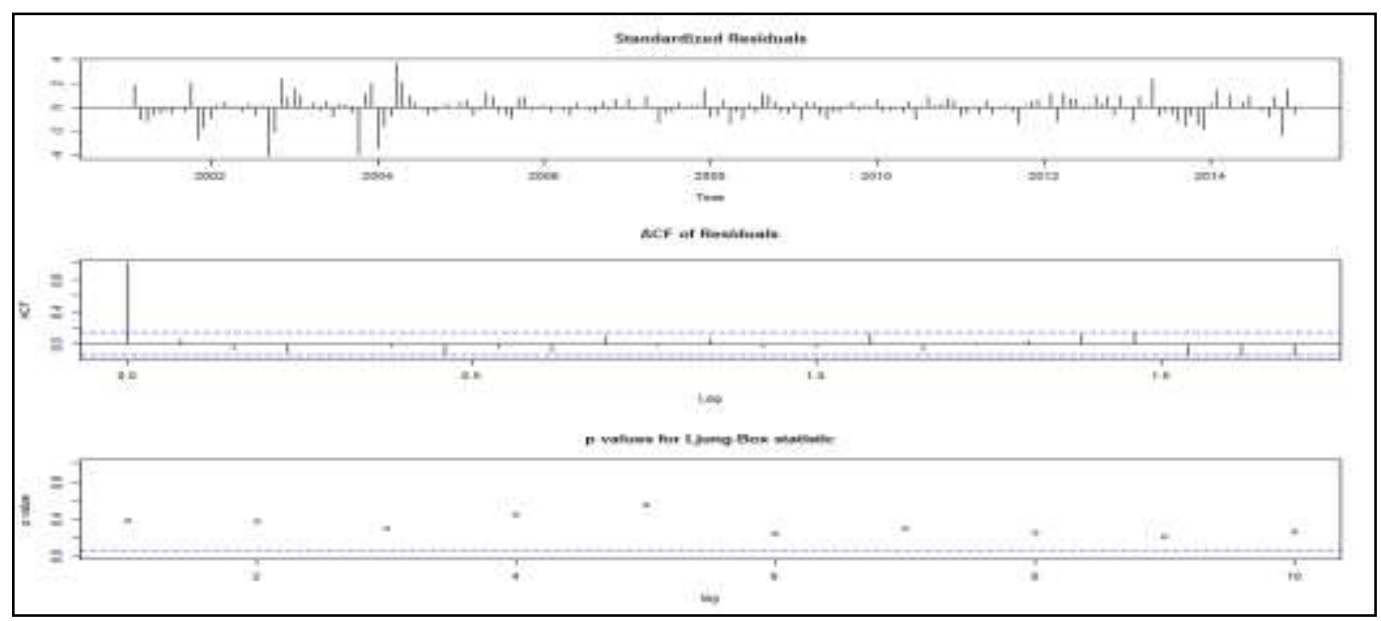

Figure 4. Diagnostic Plots for Age Less than One, (20-24) and (25-34) Years Old

\subsection{Performance Measures}

To evaluate the performance between ARIMA and State Space Model, MSE for each model have been calculated. Instead of further analyzing the data, we limit our analysis on female group age between 2534 only. This is due to the highest variation shown by the group. Forecast on the variation of number of bed admission among female age between 25-34 were done by constructing the model based on data period of 2004-2014, and comparing forecast values for data period of 2015. Table 4 shows the comparison of MSE between the two models. State Space model outperforms ARIMA due to its lowest MSE. Thus, the state space model is used for forecasting purpose.

Table 4. Comparison of MSE

\begin{tabular}{lc}
\hline Models & Value of MSE \\
\hline ARIMA & 1894.83 \\
State Space & 834.33 \\
\hline
\end{tabular}

\section{CONCLUSION}

This study analyzes the variations in number of hospital bed admission between age groups of different genders using ARIMA and State Space models. The findings from the study show that age group female of 25-34 years old have the highest values variation of total hospital bed admission. From the results, female with age within 25 until 34 years old are more unpredictable for their health. It seems true because if we compare between male and female, the latter always face or experience physical symptoms which occur much less frequently in male because of the normal patterns of fluctuation during their menstrual cycle. Related with age of 25 until 34 years old, when person grows up to 30 years old above, their body is changing bit by bit and these changes are a normal part of growing older. When person is reach to prime age of 40 and above, we can assume they can have diseases because of age factors. But for person with age 25-34 years old, we cannot assume or predict generally they will have diseases or not because it depends on the individual lifestyle. Each person has different lifestyle from their nutrition, diets, exercise and many others, so it depends on the person itself whether easy to get diseases or not. Generally, when women reach to age 30 above, their metabolism became slow and bone loss begins at this age, lead to the bone-thinning disease known as osteoporosis later in life. The unhealthy nutritional factors also lead to the diseases such as high blood pressure, high cholesterol, breast diseases and many others. In a State Space model, the variation which shows the highly varied is selected to be used in the next forecasting process. In case of total bed admission for this study, age group 25-34 years old shows the highest variation which means that total of bed admission for this group is unpredictable. The forecasted variation results from the model produce the results which approximate or similar to the actual variation from dataset of total bed admission.

However, the admission of patients into a hospital is not necessarily due to illness, it can be due to giving birth and other reasons. Hence, more research that consider other factors and other relevant methods [12] that affect the admission into a hospital should be done. 


\section{REFERENCES}

[1] Earnest, A., Chen, M. I., Ng, D., \& Sin, L. Y. Using autoregressive integrated moving average (ARIMA) models to predict and monitor the number of beds occupied during a SARS outbreak in a tertiary hospital in Singapore. BMC Health Services Research. 2005; 5(1), 36.

[2] Reis, B.Y. \& Mandl, K.D. Time Series Modeling for Syndromic Surveillance. BMC Medical Informatics and Decision Making. 2003; 3(1), pages 1-11

[3] Rahim, N. A. A., Md. Ghani, N.A., Ramli, N.M., Hashim, H. \& Musirin, I. The Application of Modified Least Trimmed Squares with Genetic Algorithms Method in Face Recognition. Indonesian Journal of Electrical Engineering and Computer Science (IJEECS). 2017; 8(1), 154-158.

[4] Umesh, I. M, Srinivasan, G.N. \& Torquato, M. Software Aging Forecasting Using Time Series Model Software Aging Forecasting Using Time Series Model. Indonesian Journal of Electrical Engineering and Computer Science (IJEECS). 2017; 8(3), 589-596.

[5] McManus, M. L., Long, M. C., Cooper, A., \& Litvak, E. Queuing theory accurately models the need for critical care resources. The Journal of the American Society of Anesthesiologists. 2004; 100(5), 1271-1276.

[6] Sinreich, D., \& Marmor, Y. Emergency department operations: the basis for developing a simulation tool. IIE transactions. 2005; 37(3), 233-245.

[7] De Bruin, A. M., Koole, G. M., \& Visser, M. C. Bottleneck analysis of emergency cardiac in-patient flow in a university setting: an application of queueing theory. Clinical and investigative medicine. 2005; 28(6), 316.

[8] Kokangul, A. A combination of deterministic and stochastic approaches to optimize bed capacity in a hospital unit. Computer methods and programs in biomedicine. 2008; 90(1), 56-65.

[9] Griffiths, J. D., Knight, V., \& Komenda, I. Bed management in a critical care unit. IMA Journal of Management Mathematics. 2013; 24(2), 137-153.

[10] Honaker, J., King, G., \& Blackwell, M. Amelia II: a program for missing data. Journal of Statistical Software. $2011 ; 45(7), 1-47$

[11] Takahashi, M. Multiple Ratio Imputation by the EMB Algorithm, Conference Of European Statisticians Work Session on Statistical Data Editing (Budapest, Hungary, 14-16 September 2015), Working Paper 34.

[12] Tayyab, M., Zhou, J., Adnan, R. \& Zahra, A. Monthly Precipitation Trend Analysis by Applying Nonparametric Mann- Kendall (MK) and Spearman's rho (SR) Tests In Dongting Lake, China: 1961-2012. Indonesian Journal of Electrical Engineering and Computer Science (IJEECS). 2017; 5(1), 41-47. 